170

\section{The Disorders of Balance in Contents between Eicosanoids in Severe Hemorrhagic Shock (SHS) as Initiating Factor in Development of Irreversibility of Process}

Oborin $A N$

Research Institute of Hematology

Lvov, Ukraine

Objective: Recognizing the important role of eicosanoids in regulatory functions of physiological systems of organisms.

Methods: The progression of severe hemorrhagic shock (SHS) in changes of prostaglandins (PGs) $\mathrm{I}_{2}, \mathrm{~F}_{2}$ a, and thromboxane (TX) $A_{2}$ content was studied in the vena cava caudalis of 10 dogs. The SHS was caused by momentary jet hemorrhage from a femoral artery (blood loss volume was $28.2 \pm 2.7 \mathrm{ml} / \mathrm{kg}$ ). The mean duration of the animals' lives after the hemorrhage was 298.6 \pm 65.9 minutes ( $\mathrm{min})$.

Results: Instantly, after the hemorrhage, the contents of PG-I $\mathrm{I}_{2}$, PG-F ${ }_{2}$, and TX-A $\mathrm{A}_{2}$ increased by $30 \%(p>.05), 2.6 \%(p>.5)$, and $96 \%(p>.001)$, respectively. After $165.7 \pm 32.0 \mathrm{~min}$ of the hemorrhage, the concentrations of PG- $\mathrm{I}_{2}, \mathrm{PG}-\mathrm{F}_{2}$ and TX-A exceeded the initial levels by $87.3 \%, 73.5 \%$ and $129.6 \%$ ( $p$ $<.001)$ respectively. However, after $278.5 \pm 50.8 \mathrm{~min}$ of the hemorrhage, the content of $\mathbf{P G I}_{2}$ exceeded the initial levels only by $87.4 \% \quad(p<.001)$, and then the concentrations of PG-F and TX- $\mathrm{A}_{2}$ were higher by $95.2 \%$ and $153.7 \%(p<.001)$ respectively.

Conclusions: $T X-A_{2}$ and $P G-F_{2}$ increases at the first stages of shock progression is one of the mechanisms of protective reaction directed to stop bleeding. However, the growing content of these eicosanoids not only opposed the cytoprotective effects of $\mathrm{PG}-\mathrm{I}_{2}$ action, but promotes severe disorders of coronary circulation. Besides, TX-A $\mathrm{A}_{2}$ and $\mathrm{PG}-\mathrm{F}_{2}$ potentiate the effects of platelet activating factor and leukotrienes, and initiate the processes of lipid peroxidation.

\section{1}

\section{Autologous Transfusion}

Gasparine Neto SG, de Almeida Silva E, Reis CG, Barbosa MA, Pinheiro P

Hospital Municipal Miguel Couto

Rio de Janeiro, Brazil

The authors present a simplification of an auto-hemotransfusion technique, used preferentially in patients presenting to chronic intensive care (CIC) with trauma to hemothorax.

During the period from January 1988 through June 1992, 42 patients have been treated with the technique. Five of them are deceased, none of the deaths being related directly to the treatment. Since it uses standard equipment, the materials employed can be found easily in any emergency ward. The method can be used for patients with hemoperitoneum using an aspiration line. The use of auto-hemotransfusion can be applied universally and largely has been used in trauma units, during prehospital assistance, in emergency wards, and during surgeries. Its main advantages are quickness, disposability, and low cost.

\section{2}

\section{Study of Abdominal Trauma Patients: Focusing on Patients with Liver or Spleen Injuries}

Tabuchi $T$, *Sato $S$, Nakata I, ${ }^{*}$ Soma $T$, Nakano $T * *$

* Department of Surgery

** Department of Internal Medicine

Tokyo Medical College, Kasumigaura Hospital

Japan

Purpose: Although abdominal traumas, particularly parenchymal injuries, have been diagnosed with reasonable accuracy through computed tomography (CT) and ultra-sonography, still it is difficult to determine whether or not laparotomy should be performed. Thus, the courses of liver and spleen injuries treated with conservative methods, mainly based on CT findings, were studied.

Methods: A total of 20 patients admitted to our hospital with injuries to the liver or spleen between April 1986 and March 1991 were used as subjects.

Results: Of 11 patients suffering liver injuries treated with conservative therapy, four and seven respectively had marginal and central bleeding lesions. Marginal fluid retention disappeared within 14.7 days on average. As for central hemorrhage, regionally bleeding lesions with a clear border and diffusely bleeding lesions were observed in two and five patients respectively. As for the rate of hematoma diminution, reduction by 1 $\mathrm{cm}$ required 6.8 days for diffuse hemorrhage and 10.5 days for regional hemorrhage, although the rate of hematoma diminution was not consistent throughout the entire course. For example, a hematoma decreased in maximum diameter from 14 to 9 $\mathrm{cm}$ during the first seven days, but took 40 days to diminish its diameter from 9 to $5 \mathrm{~cm}$. When patients with spleen injuries were examined on CT, excluding one undergoing spleenectomy due to a central tear detected by CT, it was revealed that the duration needed for hematoma disappearance greatly varied, from 30 days in the shortest case to 120 days in the longest 
case, despite the fact that hematomas formed around the spleen in an early period after injury were almost identical in size.

Conclusions: The natural history of injuries to the liver or spleen is defined when healed conservatively.

\section{3}

\section{Influence of Emergency Department Visits on the Behaviour of Hypertensive Patients}

Zeiner A, Hirschl MM, Schreiber W, Seidler D, Laggner AN

Department of Emergency Medicine, University of Vienna Vienna, Austria

Objective: Hypertensive urgencies are a common problem in emergency departments. Causes of hypertensive urgencies are insufficient medication, incorrect ingestion of drugs, and lifestyle. The aim of this study was to evaluate if a stay in an emergency department can change the behavior of hypertensive patients with regard to blood pressure control, medication intake, and lifestyle.

Methods: In a retrospective study, all patients were evaluated who presented with hypertensive urgencies during the last three months. Three months later, each patient received a questionnaire with the following topics: changes in therapy; frequency of blood pressure (BP) control; and behavior.

Results: Seventy-three patients (37 male, 36 female; age 56-13 years) received the questionnaire. Within three weeks, 30 (41\%) patients ( 17 male, 13 female; age $58-15$ years) returned completed questionnaires.

\begin{tabular}{lcc} 
& \multicolumn{1}{c}{ Yes } & No \\
& $\mathbf{n}(\%)$ & $\mathbf{n}(\%)$ \\
Physician visit & $28(93)$ & $2(0.7)$ \\
Therapy changes & $27(90)$ & $1(1)$ \\
Frequency of BP control & $17(57)$ & $13(43)$ \\
Behavior changes & $20(66)$ & $11(34)$ \\
$\quad$ (smoking, stress, weight) & &
\end{tabular}

Conclusion: The patients returning the questionnaire seemed to be a positive, selected group of patients. Most of them visited a physician after this event and improved their medication intake. Changes in behavior and frequency of blood-pressure control were rare. It is assumed that long-term effects on behavior of hypertensive patients cannot be established. In conclusion, an enforced information about risks and consequences of hypertension seemed to be necessary to achieve long-term effects on behavior of hypertensive patients after a visit in an emergency department.

\section{4}

\section{Standing Orders: Does This System Decrease the Prehospital Care Error Rate?}

Holliman CJ, Wuerz RC, Meador SA

The Pennsylvania State University

Hershey, Pennsylvania, USA

Objective: The aim of this study was to compare the error rates of physician medical commanders and paramedics before and after implementation of a standing-orders protocol system for patient care by paramedics.

Design: Physician review of prehospital trip sheet conducted prospectively as part of an ongoing quality assurance (QA) program.

Setting: An urban paramedic service in the northeastern United States.

Participants: A total of 2,001 advanced life support (ALS) run reports from the start date 1 April 1991 of the protocol system through 31 January 1992 were reviewed as part of the QA program.

Interventions: Errors in patient care (failure to administer an indicated treatment or medication or performing inappropriate or excessive treatment) by medical-command physicians and by paramedics were recorded. The errors were compared to the medical-command errors determined from a previous study encompassing transports from September 1988 through December 1990, at which time paramedics were required to obtain medical command for most treatments.

Results: Medical command errors decreased from $4.4 \%$ to $1.2 \%$ of runs after the standing-orders system was adopted. Paramedic error rates remained at $<0.5 \%$ in both systems. Mean paramedic on-scene time interval decreased by $68 \mathrm{sec}-$ onds with the standing-orders system.

Conclusions: Use of standing orders to direct initial patient care by paramedics resulted in a significant decrease in the treatment error rate by medical-command physicians, no change in the low paramedic error rate, and slightly decreased on-scene times. Use of standing orders may improve efficiency of prehospital care without compromising quality of patient care.

\section{5}

\section{Audit of Referral Practice to Radiological Department from an Emergency Ambulance}

Frossard M, Hirschl MM, Zeiner A, Seidler D, Roggla M, Laggner AN Department of Emergency Medicine, University of Vienna Vienna, Austria

Objective: Until now, the referral practice of an emergency department to the department of radiology had not been evaluated. The aim of this study was to work out the referral practice to the radiological department measured against the percentage of positive or negative radiological results.

Methods: The referral practice of the last six months was investigated with regard to chest $x$-ray, ultrasonography of abdomen and kidneys, and cranial axial tomography (CT) of the brain in 764 patients. 